Walwyn and Maitsholo Afr J Tradit Complement Altern Med. (20I2)
9(3S):24-26

\title{
PERSPECTIVES OF TRADITIONAL HEALTH PRACTITIONERS ON THE USE OF MICROBICIDES FOR THE PREVENTION OF HIV
}

\author{
Dr David Walwyn ${ }^{* a}$ and Boitumelo Maitshotlob \\ ${ }^{a}$ Graduate School of Technology Management, University of Pretoria, Pretoria, South Africa \\ biThemba Pharmaceuticals, Modderfontein, South Africa \\ *Email: dwalwyn@ithembapharma.com
}

\begin{abstract}
In many South African communities, Traditional Health Practitioners (THPs) are significant participants within a plural health care system. For several years, it has been argued that this role, especially in the context of HIV/AIDS, has not been fully optimised and THPs continue to operate outside the formal biomedical sector, where the latter forms the central means by which public health campaigns are delivered and implemented. In our previous research, we have shown that this separation of the biomedical and traditional sectors perpetuates a low level of understanding of HIV by THPs with adverse consequences for patients and the overall health care system. In this study we investigated whether biomedical/traditional division could be transformed through the involvement of THPs in the distribution of barrier microbicides; the latter are presently under investigation as a means of preventing HIV infection. We concluded that THPs could provide a willing and effective distribution network for the gel-based microbicides; given the large number of THPs and their patients, such a distribution strategy would ensure that microbicides are accessible and adopted relatively quickly within the target communities of the HIV prevention campaigns.
\end{abstract}

Keywords: Traditional Health Practitioners, HIV, AIDS, prevention, microbicides,

\section{Introduction}

In many South African communities, Traditional Health Practitioners (THPs) are significant participants within a plural health care system (Mngqundaniso et al., 2008 and Babb et al., 2007). For several years, it has been argued that this role, especially in the context of HIV/AIDS, has not been fully optimised (Wreford, 2005), and THPs continue to operate outside the formal biomedical sector, where the latter forms the central means by which public health campaigns are delivered and implemented. In our previous research, we have shown that this separation of the biomedical and traditional sectors perpetuates a low level of understanding of HIV by THPs with adverse consequences for patients and the overall health care system (Walwyn et al., 2010). In this study we investigated whether this division could be transformed through the involvement of THPs in the distribution of barrier microbicides; the latter are presently under investigation as a means of preventing HIV infection (for a full listing of all the prevention trials presently in progress, see www.avac.org and the review by Cutler et al. (2008)).

\section{Objectives of the Study}

The purpose of our research was to investigate whether THPs would be willing to play an active role in the promotion of methods for HIV/AIDS prevention, especially the use of the barrier microbicides. The latter are currently being developed to prevent transmission of sexually transmitted infections including HIV/AIDS. The study formed part of broader initiative to improve/broaden conventional prevention methods and assist in upgrading traditional practice with respect to HIV. The project was designed to guide the pharmaceutical sector in whether and how to engage with the traditional sector, and how to develop better approaches for attaining higher levels of cohesion and integration between the traditional and the biomedical sectors. In particular, the project looked at the following important questions:

- $\quad$ Are THPs already involved in advising patients about HIV prevention methods, including the use of condoms?

- How would THPs respond to the advent of microbicides?

- What community-level cultural factors may affect the use of microbicides?

- How would patients from disadvantaged areas access microbicides?

- Would THPs be prepared to distribute barrier gels from their practice?

- $\quad$ Would THPs be prepared to attend training programmes on microbicides?

\section{Methodology}

A total of 25 THPs, equally distributed between the provinces of Gauteng and Limpopo, were interviewed. In all cases, the participants were taken through a semi-structured interview covering an assessment of their understanding of HIV, their treatment approaches and their attitudes towards microbicides. 


\section{Walwyn and Maitsholo Afr J Tradit Complement Altern Med. (20 I 2) 9(3S):24-26}

\section{Results}

$68 \%$ of those interviewed were both interested in, and in favour of, the use of microbicides in the campaign against HIV, especially considering the inherent complications associated with the use of condoms. In particular, it was considered that female patients would welcome a product of this nature should the safety and efficacy concerns be adequately addressed. The remaining THPs were either unsure about (16\%), or opposed to (16\%), the use of microbicides.

Somewhat surprisingly, considering the possible conflict between biomedicine and traditional belief/value systems, over $72 \%$ of THPs indicated that they would be prepared to promote the use of microbicides within their practices (Figure 1), and to act as a distribution channel for the product, should this be possible within the regulatory structures (Figure 2). Some caution was expressed about possible side effects of the product, including fertility problems, cervical cancer and other sexually transmitted diseases (such as Herpes).

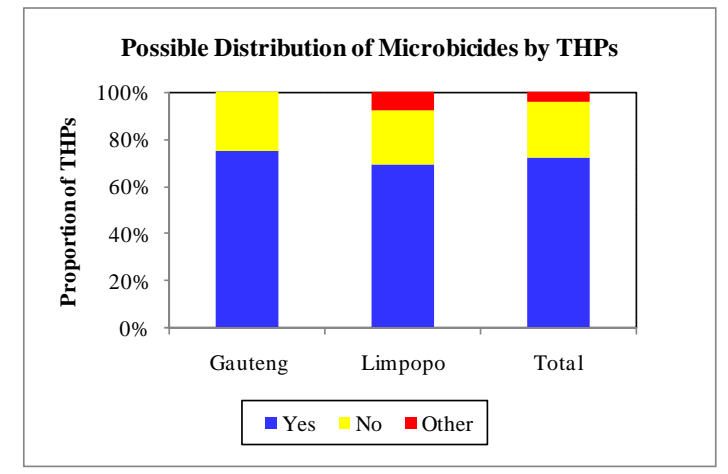

Figure 1: THPs prepared to promote use of microbicides

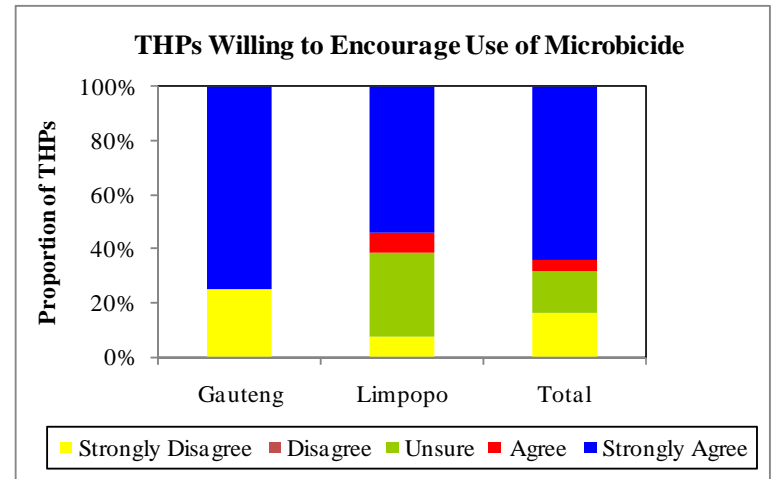

Figure 2: THPs willing to distribute microbicides

In another result, it was revealed that THPs did not believe that introduction of microbicides would encounter significant cultural resistance, despite the opinion of previously reported studies on practices such as dry sex (Tallis et al., 2005). Furthermore the THPs indicated that they would be prepared to participate in training programmes on the use of microbicides. We also noted the following points from the answers to the questionnaire:

- $\quad$ patients were counselled on the use of condoms and the latter remained the most widely used means of prevention; however the THPs perceived a general resistance to condom use especially among male clients

- $\quad$ most THPs were seeing on average 8 to $10 \mathrm{HIV}$-positive patients per month

- all patients initially assessed as HIV-positive were sent by their THPs for Voluntary Counselling and Testing(VCT); only once the diagnosis had been confirmed by the hospital or clinic was treatment initiated

- $\quad$ some THPs considered that microbicides would increase risky sexual behaviour (particularly multiple sexual partners) and their introduction would cause a decrease in condom use

- further trials may be required to establish whether substances used routinely to induce dry sex could be administered pre-coital act and in conjunction with the microbicide without affecting the latter's efficacy

- $\quad$ women were more likely than men to visit THP and to talk about HIV; the latter remained a taboo subject for many men. 


\section{Walwyn and Maitsholo Afr J Tradit Complement Altern Med. (20I2) 9(3S):24-26}

\section{Discussion}

The inclusion of THPs in the distribution channels for barrier microbicides is a matter requiring consideration and acceptance not only by the THPs themselves, but by the medicine regulatory authorities within individual countries, especially those regulations pertaining to the distribution and sale of medicines containing scheduled substances. The extent to which these changes may be required will depend on the scheduling status of the registered product. For instance, microbicides which do not include scheduled substances, such as surfactants and household bacteriostatic agents, may be registered as Schedule 0 or Schedule 1, and would normally be distributed over-the-counter through retail pharmacies and similar outlets. The presence of a registered (referred to as the responsible) pharmacist at such outlets is required before the product can be offered for sale. It is possible that new regulations to the relevant legislation (in the case of South Africa, this is the Medicines and Related Substances Control Act, 1965 (Act No 101 of 1965, as amended in 2004) (Government of South Africa, 2005).

However, microbicides such as the tenofovir-based gel, which include already registered medicines that have been previously assigned a category 3 or higher schedule, can only be dispensed by a registered pharmacists upon presentation of a doctor's prescription. The inclusion of THPs in the distribution network for a tenofovir-based microbicide would require a number of regulatory changes as follows:

- $\quad$ training of THPs in the basics of pharmaceutical dispensing

- $\quad$ registration of qualified THPs as per the intent of the Traditional Health Practitioners Act (Act No 22 of 2007)

- $\quad$ gazetting of new regulations associated with the Medicines and Related Substances Control Act, 1965 (Act No 101 of 1965) which would permit the distribution of barrier microbicides containing scheduled substances (Schedule 0 to Schedule 4) by qualified and registered THPs.

The above discussion assumes that the barrier microbicide has already been registered as a pharmaceutical product, which would of itself be a requirement for a product which claims certain health benefits and uses already registered medicinal substances. None of the changes outlined above would be impossible to implement; moreover as already mentioned, the resultant upgrading of the THPs' expertise will be of benefit to both patient and practitioner. Whilst the main objective of this research was to assess the THP response to barrier microbicides, a welcome additional affect would be the resultant value addition to THP practice.

\section{Summary and Conclusions}

With certain revisions to the medicines regulatory structure as detailed above, and the implementation of widespread training within THP structures on the use of microbicides, it is concluded that THPs could provide a willing and effective distribution network for the gel-based microbicides presently under development for the prevention of HIV. Given the large number of THPs and their patients, such a distribution strategy would ensure that microbicides could be made available and adopted relatively quickly within the target communities of the HIV prevention campaigns.

\section{Acknowledgements}

The author gratefully acknowledges the financial support of LIFElab (now part of the Technology Innovation Agency) for the project on the plant extracts and the operational costs of Arvir Technologies (within which company this research was undertaken). The research of Ms Tumi Maitshotlo on the practices of THPs in South Africa is also acknowledged.

\section{References}

1. Babb, D., Pemba, L., Seatlanyane, P., Charalambous, S., Churchyard, G. J. and Grant, A. D. (2007). Use of traditional medicine by HIVinfected individuals in South Africa in the era of antiretroviral therapy. Psychology, Health \& Medicine, 12(3): pp 314-320.

2. Cutler, B. and Justman, J. (2008). Vaginal microbicides and the prevention of HIV transmission. Lancet, 8: pp 685 - 697.

3. Government of South Africa (2004). The Medicines and Related Substances Control Acto, Act No 1010 of 1965 , as amended in 2004, available from www.info.gov.za; last accessed on $5^{\text {th }}$ December 2011.

4. Mngqundaniso, N. and Peltzer, K. (2008). Patients consulting traditional health practitioners in the context of HIV/AIDS in urban areas in KwaZulu Natal, South Africa. African Journal of Traditional, Complementary and Alternative Medicine, 5(4): pp 370-379.

5. Tallis, V., Christian, B. and Cavanagh, D. (2005). Community preparedness. Are South African communities ready for microbicides? Gender AIDS Forum, available from http://www.gaf.org.za/documents/Are\%20communities\%-20ready\%20for\%20microbicides.pdf; last accessed on $14^{\text {th }}$ June 2010.

6. Walwyn, D. and Maitshotlo, B. (2010). The role of South African Traditional Health Practitioners in the treatment of HIV/AIDS; a study of their practices and use of Herbal Medicines. Southern African Journal of HIV Medicine, 11(2); pp 11 - 17.

7. Wreford, J. (2005). Missing each other: problems and potential for collaborative efforts between biomedical and traditional healers in the time of AIDS. Social Dynamics 31(2): 55-89. 